experimentellen Untersuchungen über das mögliche Vorkommen serologisch atypischer Serumproteine bei anderen Krankheitsbildern bekannt geworden sind, die wie das Myelom mit Gewebseinschmelzungen einhergehen. Zur eindeutigen Prüfung dieser Frage und zur Klärung der möglichen Beziehung der Menge Rest-Antikörper zur Malignität des Krankheitsprozesses wird eine große Anzahl analoger immunochemischer Untersuchungen erforderlich sein.
Nach der nomenklatorischen Klassifizierung von B e n nhold ${ }^{13}$ erscheint die Zuordnung dieser individualspezifische Rest-Antikörper bildenden Myelomprotein-Komponenten zu den Paraproteinen, einer Untergruppe der Heteroproteine, gegeben.

Die Autoren danken Fräulein G. $\mathrm{H}$ a a s für die technische Assistenz bei den Stickstoffbestimmungen (K jeld a h l).

13 H. B e nnhold, Klin. Wschr. 31, 388 [1953].

\title{
Einschlußverbindungen und Verbindungen mit Einschlußbezirken in Lösung ${ }^{1}$
}

\author{
Von W. Broser und W. Lautsch \\ Aus dem Institut für Organische Chemie der Freien Universität Berlin, Berlin-Dahlem \\ (Z. Naturforschg. 8 b, 711-722 [1953]; eingegangen am 1. August 1953)
}

\begin{abstract}
Es wurde der spektrophotometrische Nachweis erbracht, daß die Cyclodextrine in Lösung mit einer Reihe von Farbstoffen nach Maßgabe des MWG. reagieren, also auch in Lösung echte Einschlußverbindungen zu bilden vermögen. Die Stabilität der Einschlußverbindungen ist um so größer, je basischer (nucleophiler) das eingeschlossene Molekül ist. Damit steht die Beobachtung in UUbereinstimmung, daß die Einschlußverbindung einer (Farbstoff-) Säure leichter Protonen abgibt, also stärker sauer ist als die freie (Farbstoff-) Säure. Diese Beobachtungen führen zu dem Schluß, daß das Cyclodextrin in seinen Einschlußverbindungen die elektrophile Komponente darstellt. - Stoffpaare, die ähnliche Eigenschaften besitzen wie die Einschlußverbindungen in Lösung, ohne jedoch echte Molekülverbindungen zu sein, enthalten Einschlußbezirke. Hierher gehören $\mathrm{u}$. a. das Addukt aus Rinderserum-Albumin und $\alpha$-Dextrin sowie die bereits früher hergestellten Hochpolymeren, die Wirkungsgruppen aus der Chlorophyll- und Häminreihe besitzen.
\end{abstract}

$\mathrm{I}_{\mathrm{i}}^{\mathrm{n}}$ m Rahmen unserer Arbeiten über Fermentmodelle ${ }^{2}$ interessierten wir uns für die Frage, ob und gegebenenfalls welche Beziehungen zwischen den von uns hergestellten ${ }^{2}$ Wirkungsgruppen aus der Chlorophyll- und Häminreihe tragenden Hochpolymeren und den Einschlußverbindungen bestehen. Die Untersuchung dieser Frage erscheint uns besonders deshalb notwendig, weil einerseits makromolekulare Substanzen, wie Polyvinylalkohol, Polyamide ${ }^{3}$ usw., aber auch Pferdemethhämoglobin ${ }^{4}$ zur Bildung von Einschlußverbindungen befähigt sind, und weil andererseits nach $\mathrm{Cr}$ a $\mathrm{m} \in \mathrm{r}^{5}$ einige in Lösung verlaufende Reaktionen in Gegenwart von Cyclodextrinen, die nach Freuden berg und $\mathrm{Cramer}{ }^{6}$ zur Bil-

1 W. La utse h u. Mitarb., 12. Mitt. über Fermentmodelle; 11. Mitt. vgl. Angew. Chem., im Druck; 1. Teilauszug der am 16.4.1953 der math.-nat. Fakultät der F.U. Berlin eingereichten Habilitationsschrift von W. B roser.

2 W. Lautsch u. Mitarbeiter, J. Polymer Sci 8, 191 [1952]; vgl. auch Kolloid-Z. 125, 72 [1952].

3 C. D. We s t, J. chem. Physics 15, 689 [1947]. dung von kristallisierten Einschlußverbindungen fähig sind, einen fermentartigen Charakter besitzen.

Nun ist aber der Begriff der Einschlußverbindung

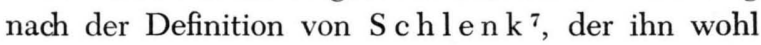
als erster vorgeschlagen hat, nur für den kristallisierten Zustand festgelegt. Es erhebt sich daher sofort die Frage, ob dieser Begriff überhaupt auf Zustände, bei denen Fermente wirksam sind, also auf Lösungen oder Kolloide, angewendet werden kann. $\mathrm{C} \mathrm{r}$ a m e r ${ }^{8}$ bejaht diese Frage in bezug auf Lösungen u. a. mit dem Hinweis auf die Tatsache, daß die Absorptionsspektren von Farbstoffen, die mit Cyclodextrinen feste Einschlußverbindungen zu bilden vermögen, in

4 M. F. P e r u tz, Trans. Faraday Soc. 42, 187 [1946]; Proc. Roy. Soc. [London] Ser. A 195, 474 [1949].

5 F. C r a m e r, Angew. Chem. 64, 626 [1952]; Liebigs Ann. Chem. 579, 17 [1953].

$6 \mathrm{~K}$. Freud e n b e rg u. F. Cra m er, Chem. Ber. 83, 296 [1950].

7 W. S chlenk jr., Fortschr. chem. Forsch. 2, 92 [1951].

8 F. Cra mer, Angew. Chem. 64, 437 [1952]. 
cyclodextrinhaltigen Lösungen verändert sind und daß Indikatoren in solchen Lösungen in anderen $p_{\mathrm{H}^{-}}$ Gebieten umschlagen als sonst. Derartige Erscheinungen werden nun aber besonders in Lösungen makromolekularer Stoffe recht häufig beobachtet. So zeigen nach $\mathrm{Michaelis}$ und $\mathrm{Granick}{ }^{9}$ viele Farbstoffe, wie Methylenblau, Toluidinblau, Kristallviolett und andere, z. B. in Agarlösungen eine neue Bande, deren Wellenlänge und Höhe von der Agarkonzentration abhängig ist. Andererseits ist bekannt, $\mathrm{da} ß$ der Umschlag von Indikatoren durch langkettige Salze (Seifen) beeinflußt wird ( $\mathrm{H}$ artley $\left.{ }^{10}\right)$. Schließlich sei noch erwähnt, daß Methylorange und ähnliche Azofarbstoffe in Gegenwart von Rinderserumalbumin eine Änderung ihres Spektrums erfahren, die - da $\gamma$-Globulin und Gelatine unwirksam sind von Klotz ${ }^{11}$ auf die Wechselwirkung der Sulfosäuregruppe des Farbstoffs mit den basischen Gruppen des Lysins und Arginins sowie auf die van der W a als schen Kräfte zwischen den aromatischen Ringen des Farbstoffs und dem Protein zurückgeführt wird.

Soll man nun auch diese Effekte mit der Bildung von Einschlußverbindungen in Zusammenhang bringen? Dies würde zweifellos zu einer Verwaschung des Begriffs führen; andererseits sind die bei den zuletzt erwähnten Fällen auftretenden Effekte nicht weniger deutlich als diejenigen, die $\mathrm{Cramer}$ veranlaßt haben, von Einschlußverbindungen in Lösung zu sprechen.

Es erscheint uns daher angezeigt, einerseits von Einschlußverbindungen in Lösungen nur dann zu sprechen, wenn der Nachweis des Vorliegens einer echten Molekülverbindung erbracht ist, andererseits aber diejenigen Gebilde, die diesen ähneln, ohne jedoch als Molekülverbindung angesprochen werden zu können, als Verbindungen mit Einschlußbezirken herauszuheben.

Unter Einschlußverbindungen in Lösung wollen wir daher in Anlehnung an die B ri e g l e b sche Definition ${ }^{12}$ der Molekülverbindung Gebilde verstehen, die mit ihren Komponenten nach Maßgabe eines einfachen MWG. im Gleichgewicht stehen, und deren Existenz in erster Linie dadurch bedingt ist, daß das

9 L. M ichaelis u. S. Granick, J. Amer. chem. Soc. 67, 1212 [1945].

10 G. S. H a rtley, Trans. Faraday Soc. 30, 444 [1934].

11 I. M. K lotz, J. Amer. chem. Soc. 68, 2299 [1946].

12 G. B ri e gl e b, Zwischenmolek. Kräfte u. Molekülstruktur, Stuttgart 1937.

$13 \mathrm{~W}$. B roser u. W. L a u s c h, Naturwiss. 40, 220 [1953].
Wirtsmolekül das Gastmolekül mehr oder weniger vollständig in sich einschließt; d.h. der räumliche Bau ist von entscheidender Bedeutung für das $\mathrm{Zu}$ standekommen der Verbindungsbildung.

Unter Verbindungen mit Einschlußbezirken wollen wir Gebilde verstehen, für die die erste der obigen Forderungen nicht erfüllt ist, die also keine echten Molekülverbindungen sind, bei denen, aber infolge der besonderen räumlichen Verhältnisse, wie z. B. Mizellbildung oder Verknüpfung des „Gastmoleküls“ mit seinem makromolekularen „Wirtsmolekül“" durch eine Atombindung oder Ionenbeziehung die zweite der obigen Forderungen erfüllt ist, d. h. daß das Wirtsmolekül das Gastmolekül zumindest partiell einschließt. Hierdurch treten ähnliche energetische Verhältnisse auf wie bei den echten Einschlußverbindungen in Lösung.

In einer kurzen Mitteilung ${ }^{13}$ berichteten wir unlängst, daß das Addukt aus $\beta$-Dextrin und Kristallviolett eine echte Molekülverbindung in Lösung bildet, also als Einschlußverbindung anzusehen ist. In der vorliegenden Arbeit wird dieser Befund an weiteren Systemen erhärtet werden; ferner werden die Prototropie-Gleichgewichte verschiedener Farbstoffe in cyclodextrinhaltigen Lösungen untersucht werden. Weiterhin soll das System RinderserumalbuminMethylorange, über das bereits $\mathrm{Klotz}{ }^{11}$ berichtete, einer Prüfung unterzogen werden. Schließlich soll über Hochpolymere mit Einschlußbezirken berichtet werden, die Wirkungsgruppen aus der Chlorophyllund Häminreihe tragen.

\section{Die Einschlußgleichgewichte}

Bei den Cyclodextrinaddukten kann die zweite der oben erwähnten Definitionsbedingungen als gegeben angesehen werden. Deshalb sollen die Untersuchungen bei ihnen beginnen.

Der Nachweis des Vorliegens eines einfachen Gleichgewichts soll dadurch erbracht werden, daß die Komponenten in wechselnden Mengenverhältnissen zusammengegeben werden und geprüft wird, ob das der mutmaßlichen Reaktionsgleichung entsprechende Massenwirkungsgesetz (MWG) dabei befolgt wird. Macht man zunächst den einfachsten Ansatz, daß je ein Cyclodextrinmolekül ein Gastmolekül einschließt, so erhält man für den Dissoziationsvorgang

mit dem MWG.

$$
\mathrm{DF} \rightleftarrows \mathrm{D}+\mathrm{F}
$$

[F] [D]

$$
\frac{[\mathrm{F}][\mathrm{D}]}{[\mathrm{DF}]}=K_{\mathrm{F}},
$$


woraus weiter folgt :

$$
\begin{aligned}
p_{K_{\mathrm{F}}} & \equiv-\lg K_{\mathrm{F}}=\frac{\Delta G^{\circ}}{4,573 T}, \\
\mathrm{D} & =\text { Cyclodextrin, } \\
\mathrm{F} & =\text { Gastmolekül, } \\
\mathrm{DF} & =\text { Einschlußverbindung, } \\
\mathrm{IG}^{\circ} & =\text { Normalreaktionsarbeit. }
\end{aligned}
$$

Zur experimentellen Bestimmung der einzelnen Gleichgewichtskonzentrationen in Gl.(1) wollen wir uns zweckmäßigerweise absorptionsspektrometrischer Methoden bedienen. Durch Einführung der der Messung zugänglichen Extinktionen in Gl. (1) erhält man

$$
\frac{E-E_{\mathrm{DF}}}{E_{\mathrm{F}}-E}=\frac{K_{\mathrm{F}}}{[\mathrm{D}]} .
$$

$E$ ist die Extinktion bei der Dextrinkonzentration [D], $E_{\mathrm{DF}}$ bzw. $E_{\mathrm{F}}$ sind die Extinktionen, die gemessen werden, wenn das Gleichgewicht (I) praktisch völlig auf der linken bzw. auf der rechten Seite liegt. Da man dies durch geeignete Wahl der Bruttokonzentrationen im allgemeinen verwirklichen kann, sind diese Größen der direkten Messung zugänglich. Im allgemeinen ist bei den untersuchten Systemen das Cyclodextrin in so großem Überschuß über das Gastmolekül vorhanden, daß die Dextringleichgewichtskonzentration [D] der Bruttokonzentration $c_{\mathrm{D}}$ gleichgesetzt werden kann. Trägt man die gemessenen Extinktionswerte gegen $\log c_{\mathrm{D}}$ auf, so erhält man, wenn Gl.(1) gilt, eine S-Kurve mit sich asymptotisch den Werten $E_{\mathrm{F}}$ und $E_{\mathrm{DF}}$ nähernden Ästen. Diejenige Dextrinkonzentration, bei der $E=\left(E_{\mathrm{F}}+E_{\mathrm{DF}}\right) / 2$, stellt, wie ein Blick auf Gl. (2) zeigt, den Zahlenwert der Gleichgewichtskonstante $K_{\mathrm{F}}$ dar. Findet man, daß die Meßpunkte auf einer solchen Kurve liegen, so ist die Gültigkeit von Gl. (1) und damit die Existenz einer echten Einschlußverbindung bewiesen.

Für den Fall, daß die einfachste Annahme nach Schema (I) nicht zutrifft, sondern daß beispielsweise zwei Dextrinmoleküle mit einem Gastmolekül eine Einschlußverbindung bilden, erhält man, wie sich zeigen läßt, eine steilere S-Kurve. Man hat also die Möglichkeit, aus der Form der erhaltenen Kurven auf die jeweils vorliegende Reaktionsgleichung zu schließen.

Erhält man dagegen bei den Untersuchungen keine S-Kurven oder nur solche mit gebrochenen Exponenten der Dextrinkonzentration, so liegt kein einfaches Gleichgewicht und auch keine echte Einschlußverbindung in Lösung vor. Optische Untersuchungen der angedeuteten Art erlauben also, die aufgeworfene Frage, Einschlußverbindung oder nicht, in eindeutiger Form zu beantworten.
Die Gleichgewichtsmessungen wurden in der Weise durchgeführt, daß eine Reihe von Lösungen stets gleicher Farbstoff-Bruttokonzentrationen $\left(c_{\mathrm{F}}=10^{-5} \mathrm{Mol} / l\right)$ jedoch in weiten Grenzen wechselnden $\beta$-Dextrin-Bruttokonzen-

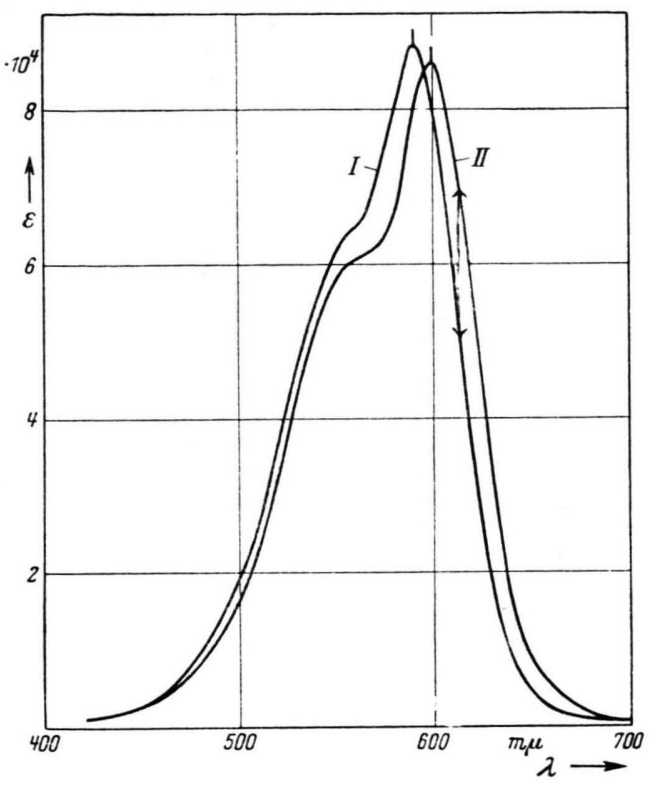

Abb. 1. Spektrum von Kristallviolett ohne (I) und mit $\beta$-Dextrin (II). $c_{\mathrm{F}}=10^{-5} ; c_{\mathrm{D}}=0,01 ; p_{\mathrm{H}} 4,95$.

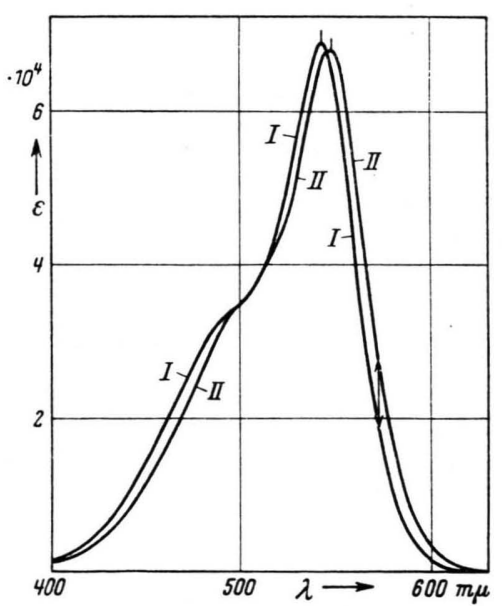

Abb. 2. Spektrum von Fuchsin ohne (I) und mit $\beta$-Dextrin (II). $c_{\mathrm{F}}=10^{-5} ; c_{\mathrm{D}}=0,01 ; p_{\mathrm{H}} 4,49$.

trationen $\left(c_{\mathrm{D}}=2 \cdot 10^{-5}\right.$ bis $\left.1 \cdot 10^{-2} \mathrm{Mol} / l\right)$ hergestellt wurde. Der gewünschte $p_{\mathrm{H}}$-Wert wurde dabei durch Zusatz stets gleicher Mengen Pufferlösung eingestellt. Zum Vergleich wurde eine Lösung ohne Dextrinzusatz hergestellt. Von dieser und von der Lösung mit dem höchsten Dextringehalt wurden die Absorptionsspektren aufgenommen. 
Man erkennt aus den Abb. 1-4, daß die Spektren durch den Cyclodextrinzusatz geringfügige Veränderungen erlitten haben. Der Unterschied der Spektren ist zur Gleichgewichtsmessung ausreichend. Die hierfür jeweils günstigsten Wellenlängen sind in den Abbildungen durch Doppelpfeile gekennzeichnet. Die $p_{\mathrm{H}}$-Werte wurden so gewählt, daß die Farbstoffe

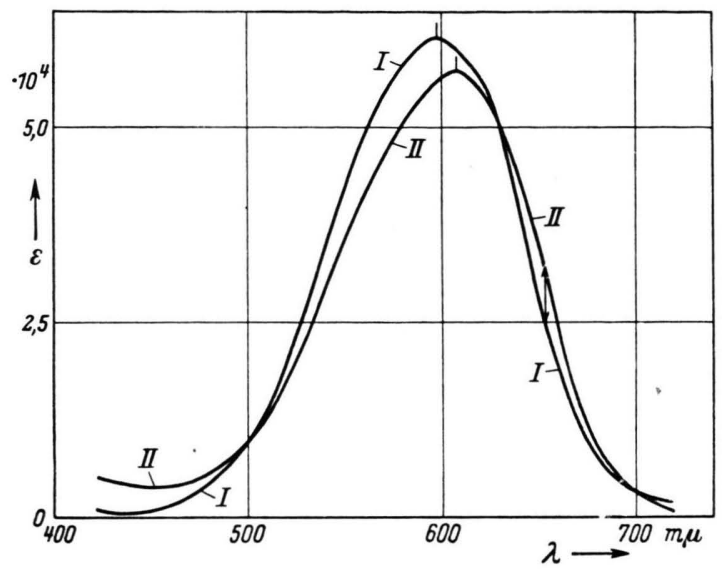

Abb. 3. Spektrum von Wasserblau ohne (I) und mit $\beta$ Dextrin (II). $c_{\mathrm{F}}=10^{-5} ; c_{\mathrm{D}}=0,01 ; 0,08-n$. HCl.

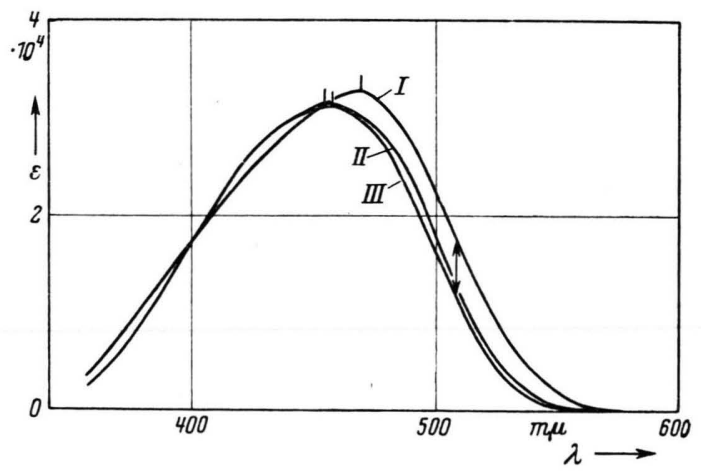

Abb. 4. Spektren von Methylorange; $c_{\mathrm{F}}=10^{-5} ; p_{\mathrm{H}} 6,08$, (I) ohne Dextrin, (II) mit $\alpha$-Dextrin, $c_{\mathrm{D}}=0,004$; (III) mit $\beta$-Dextrin, $c_{\mathrm{D}}=0,004$.

praktisch völlig in einer Form vorliegen. Im Fall des Kristallvioletts und des Fuchsins war dies das einsäurige Kation, dies trifft auch auf das Wasserblau $\mathrm{zu}$, wenn man von den Ladungen der drei Sulfophenylreste absieht. Im Falle von Methylorange lag der Farbstoff völlig als gelbes Anion vor.

In den Abb. 5-9 ist die Abhängigkeit der Extinktion von der Dextrinkonzentration wiedergegeben ( $d=$ Schichtdicke der Meßküvette). Die eingezeichneten Kreuze stellen die Meßwerte dar, während die

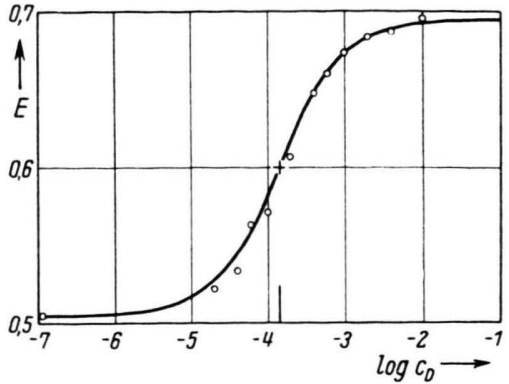

Abb. 5. Gleichgewicht $\beta$-Dextrin-Kristallviolett. $20,00^{\circ} \mathrm{C}$, $p_{\mathrm{H}} 4,95,614 \mathrm{~m} \mu, c_{\mathrm{F}}=10^{-5}, d=1 \mathrm{~cm}$.

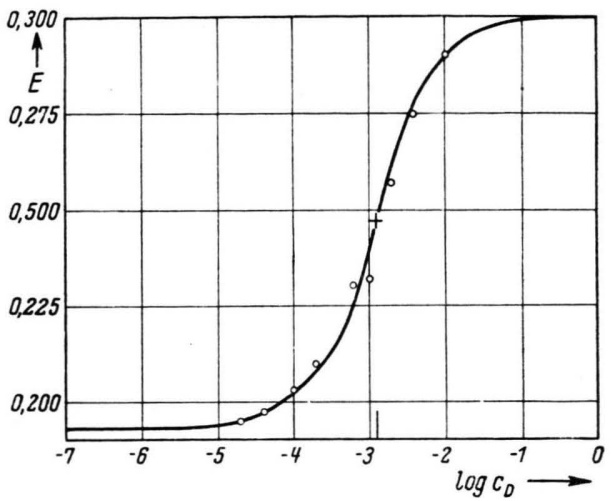

Abb. 6. Gleichgewicht $\beta$-Dextrin-Fuchsin. $20,00^{\circ} \mathrm{C}, p_{\mathrm{H}} 4,49$ $572 \mathrm{~m} \mu, c_{\mathrm{F}}=10^{-5}, d=1 \mathrm{~cm}$.

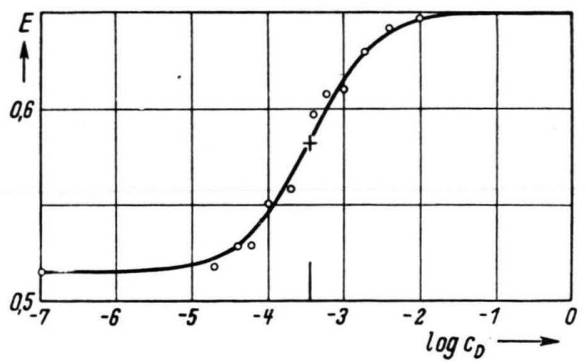

Abb. 7. Gleichgewicht $\beta$-Dextrin-Wasserblau. $20,00^{\circ} \mathrm{C}$, $0,08-n . \mathrm{HCl}, 653 \mathrm{~m} \mu, c_{\mathrm{F}}=10^{-5}, d=1 \mathrm{~cm}$.

durch sie gelegten, ausgezogenen Kurven Gl. (2) entsprechen. Damit ist das Vorliegen eines Gleichgewichtes, das dem MWG Gl. (1) und damit der Reaktionsgleichung (I) entspricht, experimentell in allen Fällen nachgewiesen. Es ist also nicht nur sichergestellt, daß die untersuchten Cyclodextrinaddukte echte Einschlußverbindungen in Lösung sind, sondern es ist damit auch bewiesen, daß ein Cyclodextrinmolekül ein Farbstoffmolekül aufnimmt. Aus den Abbildungen entnimmt man für die Gleichge- 
wichtskonstanten bei $20^{\circ} \mathrm{C}$ die in Tab. 1 wiedergegebenen Werte. Je größer $p_{\mathrm{K}_{\mathrm{F}}}$ ist, desto stabiler ist die Einschlußverbindung. Man erkennt, daß z. B. $\beta$-Dextrin-Kristallviolett stabiler als $\beta$-Dextrin-Fuchsin ist und daß die $\alpha$-Dextrineinschlußverbindung

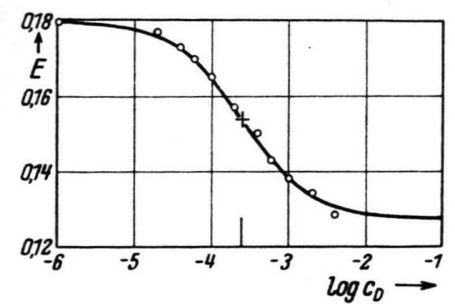

Abb. 8. Gleichgewicht $\beta$-Dextrin-Methylorange. $20,00^{\circ} \mathrm{C}$, $p_{\mathrm{H}} 6,08,508 \mathrm{~m} \mu, c_{\mathrm{F}}=10^{-5}, d=1 \mathrm{~cm}$.

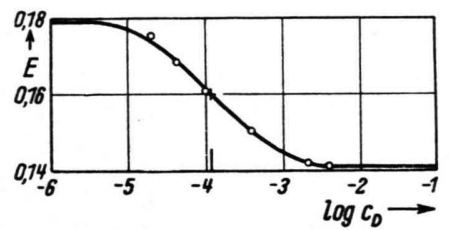

Abb. 9. Gleichgewicht $\alpha$-Dextrin-Methylorange. $20,00^{\circ} \mathrm{C}$, $p_{\mathrm{H}} 6,08,508 \mathrm{~m} \mu, c_{\mathrm{F}}=10^{-5}, d=1 \mathrm{~cm}$.

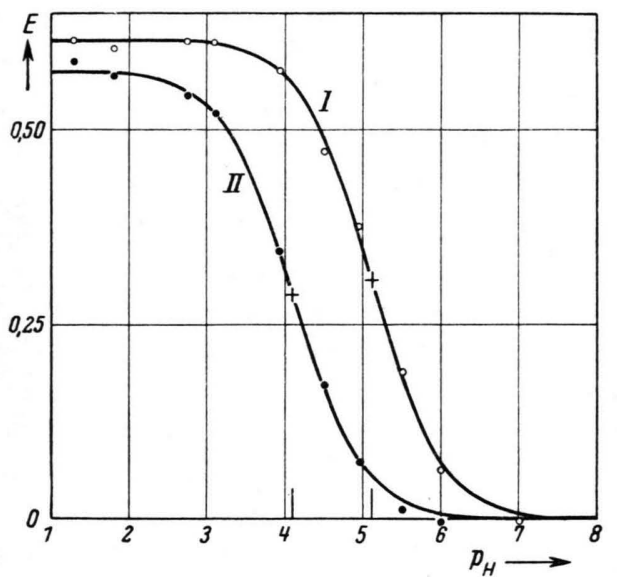

Abb. 10. Prototropie-Gleichgewichte von Wasserblau (I) und $\beta$-Dextrin-Wasserblau (II). $c_{\mathrm{F}}=10^{-5}, 20,00^{\circ} \mathrm{C}$, $c_{\mathrm{D}}=0,01,598 \mathrm{~m} \mu$ (I) bzw. $608 \mathrm{~m} \mu$ (II).

des Methylorange stabiler ist als die $\beta$-Dextrineinschlußverbindung.

2. Die Prototropie-Gleichgewichte der Einschlußverbindungen

Nach $\mathrm{Cr}$ a me ${ }^{8}$ zeigen Indikatoren noch in saureren $p_{\mathrm{H}}$-Gebieten also sonst in Cyclodextrinlösungen die „alkalische“ Farbe; Cr a m e r spricht in diesem

\begin{tabular}{|l|c|c|c|}
\hline Substanz & $\begin{array}{c}K_{\mathrm{F}} \\
\mathrm{Mol} / l\end{array}$ & $p_{\mathrm{K}_{\mathrm{F}}}$ & $\begin{array}{c}\Delta G^{0} \\
\mathrm{kcal} \\
\mathrm{Mol}\end{array}$ \\
\hline$\beta$-Dextrin-Kristallviolett. & $1,41 \cdot 10^{-4}$ & 3,85 & 5,16 \\
$\beta$-Dextrin-Fuchsin . . . & $1,26 \cdot 10^{-3}$ & 2,90 & 3,89 \\
$\beta$-Dextrin-Wasserblau . . & $3,55 \cdot 10^{-4}$ & 3,45 & 4,62 \\
$\beta$-Dextrin-Methylorange . & $2,51 \cdot 10^{-4}$ & 3,60 & 4,80 \\
$\alpha$-Dextrin-Methylorange . & $1,12 \cdot 10^{-4}$ & 3,95 & 5,25 \\
\hline
\end{tabular}

Tab. 1. Gleichgewichtskonstanten und Reaktionsarbeiten der Einschlußgleichgewichte.

Zusammenhang geradezu von „Raumalkalität“ oder „topochemischer Basizität “ in Einschlußverbindungen. Wir vermuten, daß der Grund für diese Erscheinung auf einer Vergrößerung der Säuredissoziationskonstante der Einschlußverbindung gegenüber derjenigen der freien Verbindung beruht, d. h. daß der Farbumschlag bei kleineren $p_{\mathrm{H}}$-Werten erfolgt. Zur Erhärtung dieser Vermutung muß nachgewiesen werden, daß auch in cyclodextrinhaltiger Lösung normale Prototropie-Gleichgewichte vorliegen. Dieser Nachweis soll auf spektrophotometrischem Wege zunächst am $\beta$-Dextrin-Wasserblau erbracht werden.

$\mathrm{Zu}$ diesem Zweck wurde eine Reihe von Lösungen stets gleicher Wasserblau- und $\beta$-Dextrinkonzentrationen, jedoch wechselnden $p_{\mathrm{H}}$-Wertes hergestellt. Wie man aus Abb. 7 erkennen kann, liegt bei der in der vorliegenden Versuchsreihe verwendeten $\beta$-Dextrinkonzentration das blaue Ion praktisch vollständig als Einschlußverbindung vor. Zum Vergleich wurde auch eine entsprechende Meßreihe ohne $\beta$-Dextrin untersucht. Die Extinktionsmessungen erfolgten bei den Wellenlängen der jeweiligen Absorptionsmaxima. Da der Farbumschlag von blau nach farblos langsam verläuft, wurden die Meßlösungen erst nach Erreichung des totalen Gleichgewichts, was mehrere Stunden nach der Herstellung der Fall war, vermessen.

Das Ergebnis beider Meßreihen ist in Abb. 10 dargestellt. Man erkennt, daß in beiden Fällen die Beziehungen

$$
\begin{gathered}
K_{\mathrm{FS}}=\frac{[\mathrm{FOH}][\mathrm{H}]}{[\mathrm{F}]}=[\mathrm{H}] \frac{E-E_{\mathrm{F}}}{E_{\mathrm{FOH}}-E}, \\
K_{\mathrm{DFS}}=\frac{[\mathrm{DFOH}][\mathrm{H}]}{[\mathrm{DF}]}=[\mathrm{H}] \frac{E-E_{\mathrm{DF}}}{E_{\mathrm{DFOH}}-E}
\end{gathered}
$$

erfüllt sind ${ }^{*}$. Man entnimmt Abb. 10 die Werte

$$
\begin{aligned}
p_{K_{\mathrm{FS}}} & =5,1 \text { entspr. } \quad K_{\mathrm{FS}}=7,9 \cdot 10^{-6}, \\
p_{K_{\mathrm{DFS}}} & =4,1 \text { entspr. } K_{\mathrm{DFS}}=7,9 \cdot 10^{-5} .
\end{aligned}
$$

* Die Ladungszeichen werden in den Formeln fortgelassen, soweit sie entbehrlich sind. 
Man sieht also, daß auch zwischen den verschiedenen Formen der Einschlußverbindung ein normales Prototropie-Gleichgewicht besteht, was ein weiterer Beweis für die Existenz einer echten Verbindung ist, und daß die sogenannte „Raumalkalität" eine Folge davon ist, daß DF gegenüber $\mathrm{DFOH}$ eine stärkere Säure ist als $\mathrm{F}$ gegenüber $\mathrm{FOH}$.

Nachdem an einem Beispiel der Beweis für das Vorliegen eines Prototropie-Gleichgewichtes durch Aufnahme der vollständigen S-förmigen Kurve erbracht ist, kann in den folgenden Fällen zur Ermittlung der $p_{K_{\mathrm{DFS}}}$-Werte ein vereinfachtes Verfahren eingeschlagen werden. Es sind dafür jeweils drei Messungen erforderlich. Je eine im $p_{\mathrm{H}}$-Bereich, in dem mit Bestimmtheit der Farbstoff völlig in der „sauren" bzw. ,alkalischen“ Form vorliegt, und eine bei einem $p_{\mathrm{H}}$-Wert, der in der Nähe des vermuteten Umschlagspunktes liegt.

Wendet man dieses Verfahren auf die Prototropien des Fuchsins

$$
\begin{gathered}
\mathrm{FH}^{++} \rightleftarrows \mathrm{F}_{2+}^{+}+\mathrm{H}^{+} \\
\mathrm{F}^{+}+\mathrm{H}_{2} \mathrm{O} \rightleftarrows \mathrm{FOH}+\mathrm{H}^{+}
\end{gathered}
$$

$\left(\mathrm{F}^{+}=\right.$rote Form des Fuchsins, $\mathrm{FOH}=$ Carbinolbase $)$

an und mißt bei den Wellenlängen des Absorptionsmaximums von $\mathrm{F}^{+}$, so können in beiden Fällen $E_{\mathrm{FH}}$ und $E_{\mathrm{FOH}}$ neben $E_{\mathrm{F}}$ vernachlässigt werden. Entsprechendes gilt auch für die analogen Gleichgewichte der Einschlußverbindungen.

$$
\begin{gathered}
\mathrm{DFH}^{++} \rightleftarrows \mathrm{DF}^{+}+\mathrm{H}^{+}, \\
\mathrm{DF}^{+}+\mathrm{H}_{2} \mathrm{O} \rightleftarrows \mathrm{DFOH}+\mathrm{H}^{+} .
\end{gathered}
$$

Für das Gleichgewicht (II) ermittelten B ros e r und $\mathrm{H}$ a v e m a n n ${ }^{14} p_{\mathrm{KFHS}} 1,48$, für das Gleichgewicht (III) ergab ein Kontrollversuch $p_{\mathrm{KFS}}$ 7,60. Da die entsprechenden $p_{\mathrm{Ks}}$-Werte von (IV) und (V) voraussichtlich etwas kleiner sein werden, wurden zwei Meßlösungen hergestellt, die durch Puffer bzw. Salzsäurezusatz auf die $p_{\mathrm{H}}$-Werte 7,07 und 1,19 gebracht wurden, im übrigen jedoch $10^{-5}-\mathrm{m}$. an Fuchsin und 0,01-m. an $\beta$-Dextrin waren. Die Messungen wurden beim isobestischen Punkt des freien Farbstoffs und der Einschlußverbindung, d. h. bei $546 \mathrm{~m} \mu$ (vgl. Abb. 2) vorgenommen. Daher konnte zur Ermittlung von $E_{\mathrm{F}}=E_{\mathrm{DF}}$ eine $10^{-5}$ - $m$. Farblösung bei $p_{\mathrm{H}} 4,5$ benutzt werden. Da sich das Gleichgewicht (IV) sofort einstellt, jedoch im Laufe der Zeit durch die Bildung des farblosen dreiwertigen Carbinolkations gestört wird, wurde die Extinktion der Lösung des $p_{\mathrm{H}} 1,19$ unmittelbar nach Zusammengeben der Komponenten in Ab-

\begin{tabular}{|c|c|c|c|}
\hline & Prototropie-Gleichgewicht & $p_{\mathrm{K}_{\mathrm{S}}}$ & $\begin{array}{c}K_{\mathrm{S}} \\
\mathrm{Mol} / l\end{array}$ \\
\hline \multirow{2}{*}{ 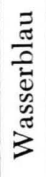 } & $\begin{array}{l}\text { ohne Dextrin } \\
\mathrm{F}^{\oplus}+\mathrm{H}_{2} \mathrm{O} \rightleftarrows \mathrm{FOH}+\mathrm{H}^{\oplus}\end{array}$ & 5,10 & $7,90 \cdot 10^{-t}$ \\
\hline & $\begin{array}{l}\text { mit } \beta \text {-Dextrin } \\
\mathrm{DF}^{\oplus}+\mathrm{H}_{2} \mathrm{O} \rightleftarrows \mathrm{DFOH}+\mathrm{H}^{\oplus}\end{array}$ & 4,10 & $7,90 \cdot 10^{-5}$ \\
\hline \multirow{4}{*}{ 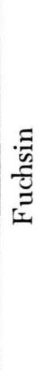 } & $\begin{array}{l}\text { ohne Dextrin } \\
\mathrm{FH}^{\oplus \oplus} \stackrel{\mathrm{DF}}{ }^{\oplus}+\mathrm{H}^{\oplus}\end{array}$ & 1,48 & $3,32 \cdot 10^{-2}$ \\
\hline & $\begin{array}{l}\text { mit } \beta \text {-Dextrin } \\
\mathrm{DFH}^{\oplus \oplus} \rightleftarrows \mathrm{DF}^{\oplus}+\mathrm{H}^{\oplus}\end{array}$ & 1,13 & $7,42 \cdot 10^{-2}$ \\
\hline & $\begin{array}{l}\text { ohne Dextrin } \\
\mathrm{F}^{\oplus}+\mathrm{H}_{2} \mathrm{O} \rightleftarrows \mathrm{FOH}+\mathrm{H}^{\oplus}\end{array}$ & 7,60 & $2,52 \cdot 10^{-8}$ \\
\hline & $\begin{array}{l}\text { mit } \beta \text {-Dextrin } \\
\mathrm{DF}^{\oplus}+\mathrm{H}_{2} \mathrm{O} \rightleftarrows \mathrm{DFOH}+\mathrm{H}^{\oplus}\end{array}$ & 7,12 & $7,60 \cdot 10^{-8}$ \\
\hline
\end{tabular}
hängigkeit von der Zeit gemessen. Durch Extrapolation

14 W. B roser u. R. Have ma n n, Z. Naturforschg. 5 a, $385[1950]$.
Tab. 2. Säuredissoziationskonstanten einiger Farbstoffe und deren Cyclodextrin-Einschlußverbindungen.

auf die Zeit Null wurde der für das Gleichgewicht (IV) charakteristische Wert $E_{I V}=0,364$ ermittelt. Das Gleichgewicht (V) stellte sich dagegen langsam ein, deshalb wurde die Meßlösung des $p_{\mathrm{H}} 7,07$ erst längere Zeit nach dem Zugeben der Komponenten vermessen. Dabei ergab sich der für das Gleichgewicht (V) charakteristische Wert $E_{\mathrm{V}}=0,360$. Aus der dritten Meßlösung erhält man endlich $E_{\mathrm{DF}}=0,680$.

Aus diesen Werten errechnet sich nach Gl. (4) bzw. einer analogen Formel

$$
p_{K_{\mathrm{DFHS}}}=1,13 \text { und } p_{K_{\mathrm{DFS}}}=\mathbf{7 , 1 2} \text {. }
$$

Ein Vergleich dieser Werte mit den entsprechenden Werten des freien Farbstoffs zeigt (vgl. Tab. 2), daß die Einschlußverbindungen des Fuchsins, ebenso wie die des Wasserblaus, kleinere $p_{\mathrm{K}_{\mathrm{S}}}$-Werte besitzen als die freien Farbstoffe. Dies gilt sowohl für die Gleichgewichte zwischen den mesomeren Kationen $\mathrm{FH}^{++}$ und $\mathrm{F}^{+}$als auch zwischen letzteren und der Carbinolbase $\mathrm{FOH}$.

3. Rinderserumalbumin-Methylorange

In einer Lösung von Rinderserumalbumin ist das Spektrum der „alkalischen “ Form des Methylorange verändert, wie bereits $\mathrm{Klotz}{ }^{11}$ festgestellt hat. Eigene Messungen bestätigen diesen Befund. Abb. 11 zeigt das Spektrum des Methylorange bei $p_{\mathrm{H}} 7,08$ in 0,2-proz. Rinderserumalbuminlösung. Wie man aus einem Vergleich mit Abb. 4 erkennt, ist es dem der $\beta$-Dextrineinschlußverbindung sehr ähnlich. Die Bandenmaxima liegen in beiden Fällen bei $457 \mathrm{~m} \mu$ und 
sind gegenüber dem des freien Farbstoffs um $11 \mathrm{~m} \mu$ nach kürzeren Wellenlängen hin verschoben. Insofern ist also zwischen der Cyclodextrineinschlußverbindung und dem Albuminaddukt kein Unterschied festzustellen. Dieser macht sich jedoch bemerkbar, wenn man versucht, eine Gleichgewichtsmessung wie beim Cyclodextrin vorzunehmen.

Als Konzentrationsangabe kann hier nicht die Molarität angewendet werden, deshalb wird der Rinderserum-

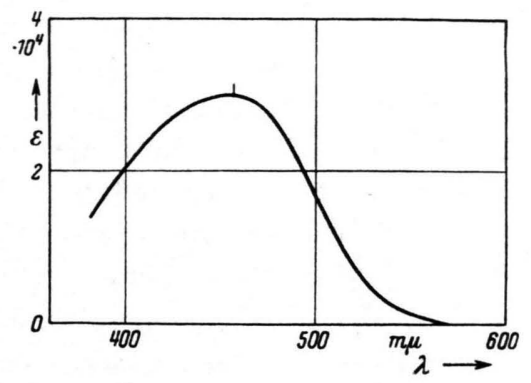

Abb. 11. Spektrum von Rinderserumalbumin-Methylorange $c_{\mathrm{F}}=10^{-5}, p=0,2 \%, p_{\mathrm{H}} 7,08$.

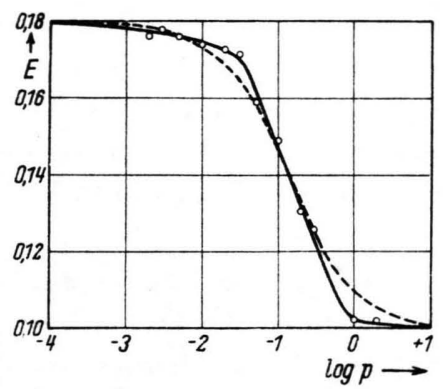

Abb. 12. Abhängigkeit der Farbstärke von Methylorange von der Konzentration $p$ des Rinderserum-Albumins.

$20,00^{\circ} \mathrm{C}, p_{\mathrm{H}} 7,08,508 \mathrm{~m} \mu, c_{\mathrm{F}}=10^{-5}, d=1 \mathrm{~cm}$.

albumingehalt in Gewichtsprozenten angegeben. Die Meßlösungen waren $10^{-5}-m$. an Farbstoff und durch Puffer auf $p_{\mathrm{H}} 7,08$ gebracht. Der Albumingehalt wurde zwischen den Grenzen 0,002\% und 2\% variiert. Die Messung der Extinktionen wurde wieder bei $508 \mathrm{~m} \mu$ vorgenommen.

In $\mathrm{Abb} .12$ sind die so gemessenen Extinktionen gegen den Logarithmus des jeweiligen Prozentgehaltes aufgetragen. Wie man erkennt, weicht die durch die Meßpunkte gelegte ausgezogene Kurve von der gestrichelten Kurve ab, die dem Verlauf beim Vorliegen eines einfachen Gleichgewichts entsprechen würde. Das Addukt Rinderserumalbumin-Methylorange entspricht also nicht den Forderungen, die an

15 H. F i s c he r u. S. G o e be l, Liebigs Ann. Chem. 524, 269 [1936]. eine Einschlußverbindung gestellt werden müssen. Da es aber in qualitativer Hinsicht den Cyclodextrineinschlußverbindungen ähnelt, wird man auf die Existenz von Einschlußbezirken schließen dürfen.

4. Hochpolymere mit Wirkungsgruppen aus der Chlorophyll- und Häminreihe

Beim Addukt Methylorange-Rinderserumalbumin ist der Farbstoff nach $\mathrm{Klotz}$ zumindest teilweise durch Ionenbeziehungen, also Hauptvalenzkräfte, an das makromolekulare Wirtsmolekül gebunden. Die hierdurch bedingt enge räumliche Nachbarschaft des Gast- und des Wirtsmoleküls verursacht die Abweichungen der Spektren. Eine andere Art, diese enge Nachbarschaft zu erzwingen, besteht darin, daß man das „Gastmolekül“" durch die andere Form der Hauptvalenz, durch die Atombindung, mit dem hochmolekularen „Wirtsmolekül“ verknüpft. Eine Dissoziation in die Komponenten ist dann natürlich ausgeschlossen. Der Nachweis, daß zusätzlich zur Atombindung eine Wechselwirkung zwischen dem "Gast-“ und „Wirtsmolekül“" statt hat, kann aus den Spektren erbracht werden. Weiter sind hier Vergleiche zwischen Prototropie- und anderen Gleichgewichten des freien und des homöopolar gebundenen "Gastmoleküls“ von Nutzen.

Nach F is cher und G o eb e ${ }^{15}$ reagiert Methylphäophorbid a mit Ammoniak und Aminen unter Sprengung des isocyclischen Ringes und Bildung der entsprechenden Säureamide. Führt man diese Umsetzung mit Polyäthylenimin durch, so erhält man, wie $\mathrm{L}$ a u t s $\mathrm{ch}^{2}$ und Mitarbeiter gezeigt haben, ein System, das aus Chloringruppen als "Gast-“ und Polyäthyleniminketten als „Wirtsmolekülen“ besteht und für das man folgende schematische Formel angeben kann.

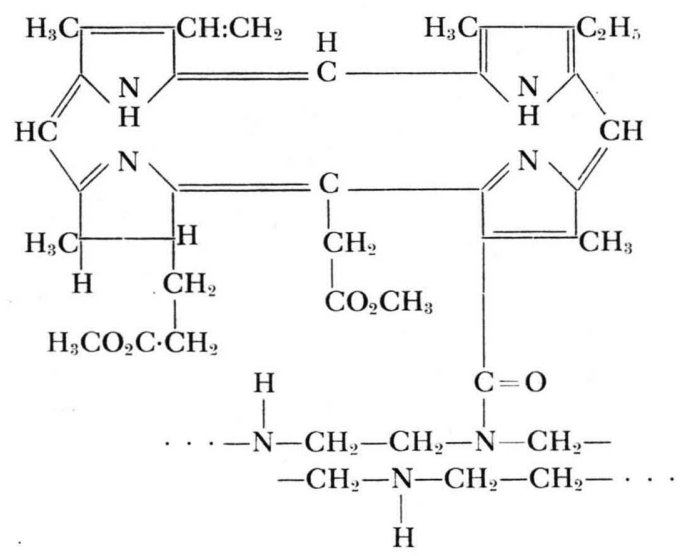


Durch geeignete Wahl der Mengenverhältnisse kann man mehr oder weniger Chloringruppen in die Polyäthyleniminketten einführen. Für die vorliegenden Untersuchungen wurde ein Produkt verwendet, das auf etwa jede hundertste Iminogruppe eine Chloringruppe enthielt.

Als niedermolekulares Analogon, also als freies „Gastmolekül“, kann man das Chlorin- $\mathrm{e}_{6}$-dimethylester-6-säureäthylamid auffassen, das man aus Methylphäophorbid a durch Behandeln mit Äthylamin erhält. Durch Vergleich der Eigenschaften dieser Verbindung mit denen des Polyäthylenimin-Derivates lassen sich Rückschlüsse über die Wechselwirkungen ziehen, die zwischen dem Chlorinrest und der Polyäthylenimin-Kette bestehen. Vergleicht man die Absorptionsspektren der verschieden hoch geladenen Formen des „Gastmoleküls“ im freien Zustand mit den entsprechenden des gebundenen Zustands, so stellt man ${ }^{16}$ fest, daß, bis auf eine charakteristische Ausnahme, eine Verschiebung der Bandenmaxima nicht zu beobachten ist. Die erwähnte Ausnahme tritt bei der langwelligen Bande des einfach geladenen Kations auf. Das Maximum dieser Bande liegt bei der niedermolekularen Verbindung bei $632 \mathrm{~m} \mu$, bei dem Polyäthylenimin-Derivat bei $644 \mathrm{~m} \mu$. Es hat hier also eine Rotverschiebung stattgefunden. Eine auf besondere Wechselwirkung zurückgehende Änderung des Spektrums ist demnach nur in diesem Fall gegeben.

Da das Chlorin durch Aufnahme von einem bzw. zwei Protonen in das ein- bzw. zweisäurige Kation übergehen kann und sich diese Übergänge im Spektrum bemerkbar machen, können Prototropie-Gleichgewichte gemessen werden. Dazu wurden Lösungen stets gleicher Chlorinkonzentration auf verschiedene $p_{\mathrm{H}}$-Werte gebracht und die Extinktionen dieser Lösungen bei geeigneten Wellenlängen gemessen. Bei der niedermolekularen Verbindung, dem freien „Gastmolekül“, ist dies bei $639 \mathrm{~m} \mu$ geschehen. Die so erhaltene Kurve ${ }^{16}$ hat zwei Wendepunkte und zwar bei $p_{\mathrm{H}} 0$ und $p_{\mathrm{H}} 2,7$. Dementsprechend beträgt $p_{\mathrm{K}_{\mathrm{FH}_{2} \mathrm{~S}}}=0$ und $p_{\mathrm{K}_{\mathrm{FHS}}}=2,7$. Die Kurve besitzt unter Berücksichtigung der gegenseitigen Überlagerung beider Gleichgewichte theoretischen Verlauf.

Bei dem hochmolekularen Produkt wurde nicht nur die $p_{\mathrm{H}}$-Abhängigkeit der Extinktion einer bestimmten Wellenlänge gemessen, sondern es wurde die $p_{\mathrm{H}}$-Abhängigkeit der ganzen langwelligen Bande aufgenommen. Dabei ergab sich ${ }^{16}$, daß mit fallendem $p_{\mathrm{H}}$ das Maximum bei $664 \mathrm{~m} \mu$ verschwindet, während dasjenige bei 644 aufgebaut wird. Der isobestische Punkt ist zwar einigermaßen angedeutet, jedoch nicht streng vorhanden. Daraus kann man schließen, daß die Änderung des Spektrums nur durch eine Umsetzung der freien Base und des einfach geladenen Kations bedingt ist, Abweichungen vom idealen Verhalten jedoch vorhanden sind. Trägt man die Extinktionen bei den beiden Wellenlängen gegen die jeweiligen $p_{\mathrm{H}}$-Werte auf, so erhält man ${ }^{16}$ zwei S-förmige Kurven, deren Wendepunkte bei $p_{\mathrm{H}}$ 3,75 liegen. Die Kurven verlaufen flacher, als es die Theorie fordert. Die Änderungen des Spektrums, die Verschiebung des Umschlagpunktes gegenüber dem des freien "Gastmoleküls“ und die $\mathrm{Ab}$ weichungen vom idealen Verhalten führen $\mathrm{zu}$ dem Schluß, daß man es auch hier mit einem System zu tun hat, das Einschlußbezirke enthält.

Eine andere Art der Schaffung einer Atombindung zwischen einem Porphinring und einer makromolekularen Kette besteht darin, Styrol und Derivate des Blatt- und Blutfarbstoffs zu copolymerisieren. Wie Broser und Lautsch ${ }^{16}$ zeigen konnten, sind deren Vinylgruppen in 2-Stellung bzw. in 2- und 4Stellung einer solchen Copolymerisierung zugänglich. Als niedermolekulare freie „Gastmoleküle“ sind hier die entsprechenden Mesoverbindungen, die also an Stelle der Vinylgruppen Äthylgruppen besitzen, aufzufassen.

Dementsprechend wurde Methylphäophorbid a einmal zum Mesomethylphäophorbid a hydriert, das andere Mal mit Styrol einer thermischen Mischpolymerisation im Molverhältnis $1000: 1$ bei $150^{\circ} \mathrm{C}$ unterworfen. Dem Polymerisat, für das man folgende schematische Formel angeben kann,

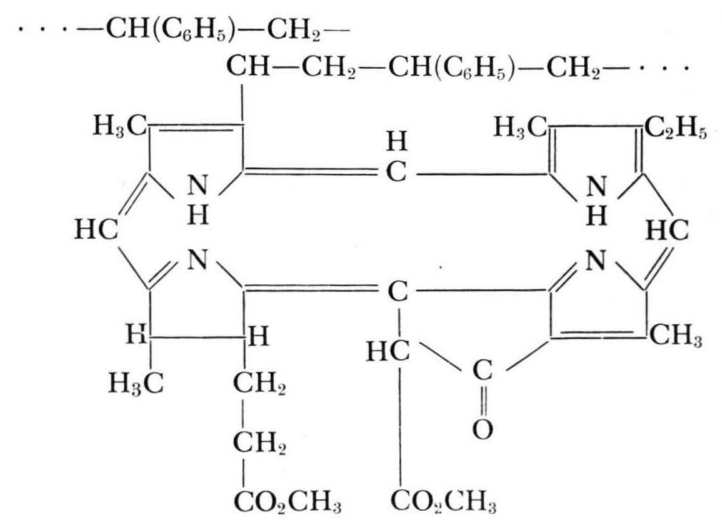

16 W. Broser u. W. La utsch, Naturwiss. 38, 208-210 [1951]. 
konnte in Toluollösung selbst mit konzentrierter Salzsäure kein Farbstoff entzogen werden. Aus den Spektren ${ }^{16}$ ist ersichtlich, daß die Banden des Polymerisats gegenüber denen des Mesomethylphäophorbids a nach Rot verschoben sind, was auf eine Wechselwirkung zwischen dem Phorbinkern und Teilen der Styrolkette schließen läßt.

Zu demselben Ergebnis gelangt man, wenn Protoporphin-dibenzylester, ein Derivat des Blutfarbstoffs, unter analogen Bedingungen mit Styrol mischpolymerisiert wird. Auch da sind die Banden des Polymeren gegenüber denen der Mesoverbindung verschoben ${ }^{16}$.

Gleichgewichtsmessungen wurden an diesen Systemen nicht vorgenommen, da sie nur in wenigen, zudem hierfür noch ungeeigneten Lösungsmitteln löslich sind. Auf Grund der Analogie mit den früheren Versuchen und auf Grund der Verschiebung der Spektren wird man aber auch bei diesen Polymerisaten mit Einschlußbezirken zu rechnen haben.

\section{Diskussion}

Die Spektren der Einschlußverbindungen der untersuchten Triphenylmethanfarbstoffe unterscheiden sich von denen der freien Farbstoffe nicht grundsätzlich. Die Banden sind jedoch durchweg etwas nach längeren Wellen verschoben und - wenn auch nur unerheblich - erniedrigt. Die Spektren des Methylorangeanions haben dagegen durch den Einschluß eine Blauverschiebung erfahren. Die Gründe hierfür sollen in der folgenden Arbeit dargelegt werden.

Betrachtet man sich die Gleichgewichtskonstanten der Bildungsreaktion der Einschlußverbindungen (vgl. Tab. 1), so stellt man fest, daß die $p_{\mathrm{K}_{\mathrm{F}}}$-Werte bei den vergleichbaren Einschlußverbindungen der $\alpha$ - und $\beta$-Dextrine bei den $\alpha$-Dextrinen größer sind; d. h. die Gastmoleküle sind im $\alpha$-Dextrin fester gebunden als im $\beta$-Dextrin. Für das Paar Fuchsin $\left(p_{\mathrm{K}_{\mathrm{FHS}}} 1,48\right)$ und Kristallviolett $\left(p_{\mathrm{K}_{\mathrm{FHS}}} 1,96\right)$ ergibt sich, daß das stärker basische Kristallviolett eine stabilere Einschlußverbindung liefert als das Fuchsin. Man kann also die Faustregel aufstellen, daß die Einschlußverbindungen um so stabiler sind, je stärker basisch oder, allgemein gesprochen, nucleophil das Gastmolekül ist. Das bedeutet aber nichts anderes, als daß das in der Einschlußverbindung enthaltene Gastmolekül den nucleophilen, das Cyclodextrin den elektrophilen Partner darstellt. Darüber hinaus führen die Untersuchungen zu dem Schluß, daß $\alpha$ Dextrin elektrophiler als $\beta$-Dextrin ist.
Die beobachtete Vergrößerung der Säuredissoziationskonstanten, d.h. die Acidifizierung durch Einschließen, führt ebenfalls zu dem Ergebnis, daß die Cyclodextrine in ihren Einschlußverbindungen die elektrophile Komponente darstellen, da sich zeigen läßt, daß die Säuredissoziationskonstanten der Einschlußverbindungen von den Einschlußgleichgewichten der „sauren “ und „alkalischen “ Formen abhängen. Wenden wir das MWG auf die Prototropie-Gleichgewichte der freien und der eingeschlossenen Farbstoffe sowie auf die für beide Formen angesetzte Reaktionsgleichung (I) an, so folgt:

$$
\begin{array}{rlrl}
K_{\mathrm{FHS}} & =\frac{[\mathrm{F}][\mathrm{H}]}{[\mathrm{FH}]} ; & (5) & K_{\mathrm{DFHS}}=\frac{[\mathrm{DF}][\mathrm{H}]}{[\mathrm{DFH}]} ; \\
K_{\mathrm{F}}=\frac{[\mathrm{F}][\mathrm{D}]}{[\mathrm{DF}]} ; & \text { (7) } & K_{\mathrm{FH}}=\frac{[\mathrm{FH}][\mathrm{D}]}{[\mathrm{DFH}]} .
\end{array}
$$

Teilen wir Gl. (5) durch Gl. (6) und Gl. (7) durch Gl. (8), so ergibt sich:

$$
\frac{K_{\mathrm{FHS}}}{K_{\mathrm{DFHS}}}=\frac{[\mathrm{F}][\mathrm{DFH}]}{[\mathrm{FH}][\mathrm{DF}]}=\frac{K_{\mathrm{F}}}{K_{\mathrm{FH}}} .
$$

$\mathrm{Da}$ in allen Fällen experimentell gefunden wurde $K_{\text {FHS }}<K_{\text {DFHS }}$, muß auch gelten $K_{\mathrm{F}}<K_{\mathrm{FH}}$; kleineres $K_{F}$ entspricht nun aber der stabileren Einschlußverbindung. Mithin ergibt die Untersuchung der Prototropie-Gleichgewichte, daß die Einschlußverbindungen der Basen $\mathrm{F}$ stabiler sind als die der korrespondierenden Säuren $\mathrm{FH}^{+}$. Das bedeutet aber, daß auch die Untersuchung der Prototropie-Gleichgewichte genau so wie die Betrachtung der Einschlußgleichgewichte zu dem Schluß führt, daß die Cyclodextrine in ihren Einschlußverbindungen die Rolle des elektrophilen Partners spielen.

Nachdem jetzt die Verhältnisse bei den Cyclodextrineinschlußverbindungen geklärt sind, wollen wir uns den Systemen mit Einschlußbezirken zuwenden.

Es wurde bereits darauf hingewiesen, daß die Veränderung, die das Spektrum des MethylorangeAnions in rinderserum-albuminhaltiger Lösung erleidet, der in cyclodextrinhaltigen Lösungen analog ist. Jedoch unterscheidet sich die Abhängigkeit der Extinktion von der Albuminkonzentration von derjenigen echter Einschlußverbindungen. Die Kurve verläuft erst flacher und dann steiler. Offenbar sind die Albuminmoleküle erst von einer bestimmten Konzentration an bereit, Gastmoleküle in verstärktem Umfang aufzunehmen.

Beim Vergleich der Spektren des Chlorin- $\mathrm{e}_{6}$-di- 
methylester-6-säure-polyäthylenimids mit denen des entsprechenden niedermolekularen Produkts fällt auf, daß eine Verschiebung nur bei dem einwertigen Kation auftritt. Dies deutet darauf hin, daß nur in diesem Fall stärkere nebenvalenzähnliche Beziehungen zwischen dem „Wirts-“ und dem „Gastmolekül“" auftreten. Nach den bei den Einschlußverbindungen gemachten Erfahrungen sollte dies ein „Umschlagen“ des Prototropie-Gleichgewichts weiter im Alkalischen zur Folge haben. Dies stimmt mit den Versuchen überein. Die niedermolekulare Verbindung schlägt bei $p_{\mathrm{H}} 2,7$, die makromolekulare bei $p_{\mathrm{H}} 3,75 \mathrm{um}$. Die

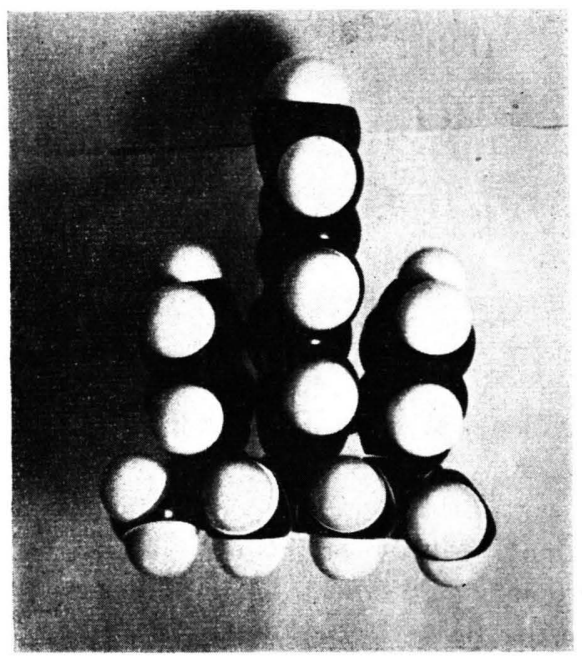

Abb. 13. Modell des Mischpolymerisates.

Abweichungen vom idealen Verhalten zeigen aber, daß wir es nicht mit einer echten Einschlußverbindung zu tun haben.

Über die Art der Wechselwirkung zwischen der Polyäthyleniminkette und der einsäurigen prosthetischen Gruppe kann man nur Vermutungen anstellen. Man wird davon auszugehen haben, daß in dem $p_{\mathrm{H}}$-Gebiet, in dem das einsäurige Kation beständig ist, alle Iminogruppen als Immoniumgruppen vorliegen, das Wirtsmolekül also elektrophil ist. Aus der beobachteten $p_{\mathrm{H}^{-}}$-Verschiebung des Umschlags folgt, daß das einsäurige Kation dann trotz seiner positiven Ladung nucleophiler als der neutrale Chlorinrest sein muß. Zum Verständnis dieser merkwürdigen Tatsache läßt sich vielleicht anführen, daß im einwertigen Kation nur noch eins der vier Ringstickstoffatome über ein einsames Elektronenpaar verfügt, die Symmetrie also gestört ist. Durch Beanspruchung dieses einsamen Elektronenpaares durch eine Protonbrücke, die von einer Immoniumgruppe des Wirtsmoleküls ausgeht, wird die Störung verkleinert. Das einsäurige Kation hätte demnach eine größere Tendenz zur Ausbildung von Protonbrücken, ist also nucleophiler als der neutrale Chlorinrest.
Was endlich die Styrolcopolymerisate betrifft, so ist auf eine auf Nebenvalenzkräfte zurückzuführende Wechselwirkung zwischen dem Wirts- und dem Gastmolekül nur auf Grund der Spektren zu schließen. Betrachtet man sich den räumlichen Bau eines solchen Polymeren am Modell (Abb. 13), so erkennt man, daß der Phorbinrest an der Kette links und rechts von Phenylresten eingeschlossen ist, und daß die Ringsysteme flach aufeinander liegen. Damit dürfte die Möglichkeit zur Wechselwirkung zwischen dem $\pi^{-}$ Elektronensystem der Benzolringe mit dem des Phorbinringes gegeben sein. Zwischen dem Gast- und dem Wirtsmolekül dürften daher in erster Linie Dispersionskräfte wirksam sein, und zwar durch die infolge Atombindung zwischen Gast- und Wirtsmolekül erzwungene enge räumliche Nachbarschaft in höherem Maße als sonst.

Die eingangs gestellte Frage nach den Beziehungen, die zwischen den Hochpolymeren, die Wirkungsgruppen aus der Chlorophyll- und Häminreihe tragen, und den Einschlußverbindungen bestehen, können wir also dahingehend beantworten, daß es sich zwar bei diesen nicht um echte Einschlußverbindungen handelt, daß aber in ihnen Bezirke existieren, in denen ähnliche Verhältnisse herrschen wie bei den Einschlußverbindungen. Darüber hinaus konnte nicht nur experimentell nachgewiesen werden, daß die Cyclodextrine echte Einschlußverbindungen in Lösung zu bilden vermögen, sondern es konnte auch gezeigt werden, daß die Cyclodextrine in ihren Einschlußverbindungen als elektrophile Komponenten fungieren.

\section{Apparaturen und Meßtechnik}

Die Messung der Absorptionsspektren und der Gleichgewichte erfolgt in einer selbstgebauten Apparatur. Nähere Einzelheiten vgl. B r o s e r und $\mathrm{H}$ a v e $\mathrm{m}$ a $\mathrm{n} n{ }^{14}$.

Die für die Untersuchung der Einschlußgleichgewichte benötigten Meßlösungen wurden wie folgt hergestellt: Je $1 \mathrm{ml}$ einer $5 \cdot 10^{-5}-m$. Stammlösung des betreffenden Farbstoffs wurde mit je $2 \mathrm{ml}$ des betreffenden Puffers bzw. 0,2-n. $\mathrm{HCl}$ mit $x \mathrm{ml}$ einer 0,01-m. Cyclodextrinlösung und mit $(2-\mathrm{x}) \mathrm{ml}$ Wasser versetzt. Da es sich herausstellte, daß es selbst bei Verwendung der besten und gründlichst gereinigten Pipetten nicht möglich war, stets ausreichend genau gleiche Mengen der Farbstofflösungen einzupipettieren, wurde die einpipettierte Farbstofflösung sowie die Gesamtmenge der Lösung durch Wägung festgestellt und daraus die genaue Farbstoffkonzentration der Meßlösung berechnet. Die gemessenen Extinktionen wurden dann rechnerisch auf den Sollwert $10^{-5}-\mathrm{m}$. korrigiert. Die konzentriertesten Cyclodextrinlösungen wurden wie oben mit $x=0$ und direkter Einwage des festen Cyclodextrins hergestellt. 
Die für die Untersuchungen der Prototropie-Gleichgewichte benötigten Meßlösungen wurden in der Regel wie folgt hergestellt: Je $1 \mathrm{ml}$ einer $5 \cdot 10^{-5}-m$. Farbstoffstammlösung wurde mit $2 \mathrm{ml}$ Wasser und $57 \mathrm{mg} \beta$-bzw. $49 \mathrm{mg} \alpha$-Dextrin (in einigen Fällen auch mit $2 \mathrm{ml}$ der 0,01- $m$. Cyclodextrinlösung) und mit $2 \mathrm{ml}$ Pufferlösung des jeweiligen $p_{\mathrm{H}}$-Wertes versetzt. Die genaue Farbstoffkonzentration wurde wieder durch Wägung ermittelt. Die Pufferlösungen hatten die Ionenstärke 0,2, die Meßlösungen daher die Ionenstärke 0,08. Die $p_{\mathrm{H}}$-Werte der Meßlösungen wurden nach Verdünnen mit Wasser im Verhältnis der Meßlösungen mit der Wasserstoffelektrode potentiometrisch bei $20,00^{\circ} \mathrm{C}$ gemessen.

\section{Die verwendeten Substanzen}

Kristallviolett. Käufliches Kristallviolett wird in viel Wasser gelöst. In die siedende Lösung gibt man so lange in kleinen Portionen 1- $n$. Natronlauge, bis die Carbinolbase ausflockt. Der Niederschlag wird abgesaugt, mit Wasser gewaschen und getrocknet. Dann wird zweimal aus Ligroin umkristallisiert. Farblose, sich an der Luft schnell violett färbende Kristalle. Schmp. $219^{\circ}$ C. - Diese Carbinolbase diente zur Herstellung der Kristallvioletturlösungen, indem sie in Wasser, das durch ganz geringen Pufferzusatz auf $p_{\mathrm{H}}$ 4,5 gebracht worden war, im gewünschten Mengenverhältnis gelöst wurde.

Fuchsin. Käufliches Diamantfuchsin wird mit 1-n. Natronlauge etwa 1 Stde. rückgekocht, die hellfarbige Lösung noch heiß durch ein Filter gegeben. Die nach dem Erkalten ausgeschiedenen farblosen Rosanilinkristalle werden rasch abgenutscht, mit verdünnter Natronlauge gewaschen und dann in wenig Wasser, dem etwas Salzsäure zuzugesetzt ist, aufgenommen. Aus dieser Lösung wird mit Natronlauge, wie oben, erneut Rosanilin gefällt. Die nach zweimaliger Wiederholung des Verfahrens erhaltenen Rosanilinkristalle werden im Vakuum über Natriumhydroxyd bei etwa $50^{\circ} \mathrm{C}$ scharf getrocknet. Das so erhaltene Rosanilin diente zur Herstellung der Farbstoffurlösung, die durch geringen Pufferzusatz auf $p_{\mathrm{H}}$ 4,5 eingestellt wurde.

Wasserblau. Es wurde Wasserblau 6 B extra $\mathrm{P}$ von B a y e r, Leverkusen, direkt verwendet. Nach Angabe der Herstellerfirma handelt es sich dabei um das Dinatriumsalz des $N, N^{\prime}, N^{\prime \prime}$-Tri-(4-sulfophenyl)- $p, p^{\prime}$-diaminofuchsonimmonium. Wie die Versuche ergaben, ist es für die benötigten Zwecke hinreichend rein. Durch Lösen der entsprechenden Menge dieser Substanz in reinem Wasser wurden die Urlösungen hergestellt.

Methylorange. Käufliches Methylorange wurde wiederholt aus Wasser umkristallisiert. Wie die Versuche ergaben, ist es hinreichend rein. Als Urlösung dienten entsprechend konzentrierte wäßrige Lösungen.

Rinderserumalbumin. Es wurde Rinderserumalbumin reinst der Behring-Werke, Marburg, verwendet.

Cyclodextrine* $^{*}$. Die Cyclodextrine wurden nach der

* Die Herstellung der Cyclodextrine erfolgte durch die Herren cand. chem. H. L e h m a n n u. R. Wi e c h e r t.

$17 \mathrm{~K}$. Freudenberg, E. Plankenhorn u. H. K n a ber, Liebigs Ann. Chem. 558, 1 [1945]. von Freudenberg, Plankenhorn und Kna b $\theta \mathbf{r}^{17}$ angegebenen Arbeitsweise durch Abbau der Kartoffelstärke mit bacillus macerans (7069 America Type Culture Collection) hergestellt. Die dort angegebenen Ausbeuten wurden zu etwa $80 \%$ erreicht. Die isolierten Dextrine wurden zur Entfernung des Kristallwassers bei 1 Torr und $110^{\circ} \mathrm{C}$ über $\mathrm{P}_{2} \mathrm{O}_{5}$ bis zur Gewichtskonstanz getrocknet. Die Produkte wurden durch die spezifischen Drehungen charakterisiert. Diese stimmten innerhalb der Fehlergrenzen mit dem von $\mathrm{F}$ re u d e nb e r g angegebenen $[\alpha]_{D}=149,0 \pm 1$ für $\alpha$-Dextrin und $[\alpha]_{\mathrm{D}}=158,8 \pm 1$ für $\beta$-Dextrin überein.

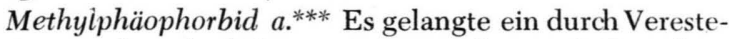
rung von Phäophorbid a mit Diazomethan nach $\mathrm{F}$ is c her und $\mathrm{S}$ p i e g e l ber g e 18 hergestelltes Produkt zur Anwendung. Schmp. $228^{\circ} \mathrm{C}$.

Mesomethylphäophorbid $a .^{* *}$ Es wurde ein Produkt verwendet, das nach $\mathrm{F}$ i s c h e r und $\mathrm{L}$ a k a t o s ${ }^{\mathbf{1 9}}$ aus Phäophorbid a durch Hydrierung mit Palladium als Katalysator und anschließender Veresterung des erhaltenen Mesophäophorbids a mit Diazomethan gewonnen wurde. Schmp. $218^{\circ} \mathrm{C}$.

Protoporphyrindibenzylester und Mesoporphyrindibenzylester $^{* *}$ wurden nach der Phosgenmethode von $\mathrm{Fi}$ $\mathrm{s} \mathrm{cher}$ und $\mathrm{S} \mathrm{ch} \mathrm{mid} \mathrm{t} 20$ hergestellt.

Chlorin- $e_{6}$-dimethylester-6-säureäthylamid.*** Methylphäophorbid a wird mit 8-proz. acetonischer Äthylaminlösung im Bombenrohr etwa $24 \mathrm{Stdn}$. auf 50-60 erwärmt. Der Inhalt des Bombenrohres wird in Äther aufgenommen und durch Waschen mit Wasser und verdünnter Natronlauge vom Aceton und verseiften Anteilen befreit. Die entstandene Verbindung wird mit 4-proz. Salzsäure ausgezogen und sofort wieder in frischen Äther übergeführt. Bei vorsichtigem Einengen kristallisiert die Verbindung in Form von blauschwarzen, würfelartigen Kristallen aus. Ausbeute $20 \%$.

$$
\begin{aligned}
& \mathrm{C}_{38} \mathrm{H}_{45} \mathrm{O}_{5} \mathrm{~N}_{5} \text { : Ber. N 10,82; Gef. N 11,03. } \\
& \text { Mol.-Gew. 651,78. }
\end{aligned}
$$

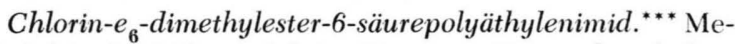
thylphäophorbid a wird in Dioxan gelöst und auf dem Wasserbad bei $60^{\circ}$ unter dauerndem Umschwenken zu einer absolut wasserfreien Lösung von Polyäthylenimin gewünschter Konzentration in Dioxan gegeben. Das Molverhältnis des Farbstoffs zum Polyäthyleniminmolekül kann in den Grenzen 1:10 bis 1:1000 variiert werden. Nach etwa 1/2 Stde. ist die Umsetzung beendet, was am Auftreten des Chlorinspektrums erkennbar ist. Das Dioxan wurde nahezu verdampft und durch Wasser ersetzt. Das Polymere war wasserlöslich und wurde in diesem Zustand untersucht.

Copolymerisation von Styrol und Methylphäophorbid a.

$20 \mathrm{mg}$ Methylphäophorbid a werden in $5 \mathrm{ml}$ frisch destillierten Styrols gelöst und im geschlossenen Wäge-

$18 \mathrm{H}$. F is che r u. G. S p i e g e l be r g e r, Liebigs Ann. Chem. 515, 130 [1935].

$19 \mathrm{H}$. F i s cher u. E. L a k a to s, Liebigs Ann. Chem. 506, 123 [1933].

20 H. Fischer u. W. Schmidt, Liebigs Ann. Chem. 519, 244 [1935]. 
glas im Trockenschrank 2 Stdn. lang auf $150^{\circ} \mathrm{C}$ erhitzt. Die nach dem Erkalten feste Masse wird in Toluol gelöst und mit Methanol gefällt. Der dabei ausfallende kautschukartige Klumpen wird zerkleinert und in reinem Methanol wiederholt entquollen. Die so erhaltenen Schnitzel stellen nach dem Trocknen eine schmutziggrüne, opake hornartige Masse dar. - Einer toluolischen Lösung des Copolymerisats kann auch mit konzentrierter Salzsäure kein Farbstoff entzogen werden. In einem Kontrollversuch wurden Polystyrol und Methylphäophorbid a in Toluol gelöst. Diese Lösung konnte durch Behandeln mit 18-proz. wäßriger Salzsäure völlig entfärbt werden. Damit ist bewiesen, daß der Farbstoff einpolymerisiert ist. Durch Viskositätsmessungen in Toluol konnte das Molekulargewicht des Copolymerisats nach der Sta u dingerschen Regel zu etwa 40000 abgeschätzt werden.
Copolymerisation von Styrol und Protoporphyrindibenzylester. $10 \mathrm{mg}$ Protoporphyrindibenzylester werden in $15 \mathrm{ml}$ Styrol gelöst und 2 Stdn. lang auf $150^{\circ}$ erhitzt. Das Polymerisat wird in Toluol gelöst und, wie vorstehend beschrieben, mit Methanol gefällt und entquollen. Die erhaltenen Schnitzel sind blaß rosa gefärbt. Einer toluolischen Lösung konnte auch hier mit konz. Salzsäure kein Farbstoff entzogen werden.

Für die freundliche Überlassung des Rinderserumalbumins sind wir den Behring-Werken in Marburg sehr verbunden. Frl. Lehmann und Frl. S e if ert danken wir für ihre Mithilfe bei der Durchführung der Messungen.

** Hergestellt von H. Zos chke.

*** Vgl. Diplomarbeit U. Döring.

\title{
Cyclodextrin-Einschlußverbindungen in Lösung ${ }^{1}$
}

\author{
Von W. Broser \\ Aus dem Institut für Organische Chemie der Freien Universität Berlin, Berlin-Dahlem \\ (Z. Naturforschg. 8 b, 722-729 [1953]; eingegangen am 1. August 1953)
}

\begin{abstract}
Es werden die Einschluß- und Prototropie-Gleichgewichte des $\alpha$-Dextrin-Methylorange und des $\beta$-Dextrin-Methylorange spektrophotometrisch untersucht. Der in der vorigen Mitteilung aufgezeigte Zusammenhang beider Gleichgewichte wird dabei verifiziert und der Schluß erhärtet, daß die Cyclodextrine in ihren Einschlußverbindungen als elektrophile Komponente fungieren. Die Änderung, die das Spektrum des Methylorange in saurer Lösung durch Cyclodextrinzusatz erfährt, wird auf die. Tautomerie zwischen der roten „Azonium“-Form und der farblosen „Ammonium“Form des Methylorangezwitterions zurückgeführt. Eine Diskussion der energetischen Verhältnisse ergibt, daß im Cyclodexrin-Hohlraum eine hohe Feldstärke herrscht, was zur Folge hat, daß Dipolmoleküle und polarisierbare Verbindungen dort festgehalten werden. Da die potentielle Energie der Polarisierbarkeit und damit der Molekularrefraktion proportional ist, geht die Stabilität der Einschlußverbindungen der Molekularrefraktion symbat. Zum Schluß wird die hier vertretene Auffassung mit derjenigen C r a m e r s verglichen.
\end{abstract}

$\mathrm{I}_{\mathrm{m}}^{\mathrm{n}}$ n der vorangegangenen Arbeit ${ }^{1}$ wurde der experimentelle Beweis erbracht, daß echte CyclodextrinEinschlußverbindungen in Lösung existieren. Es wurde weiter gezeigt, daß die bei den Einschlußverbindungen beobachtete Verschiebung der $p_{\mathrm{K}_{\mathrm{S}}}$-Werte von Prototropie-Gleichgewichten nach kleineren Werten hin darauf zurückzuführen ist, daß die Einschlußverbindung der „alkalischen“ Form des Gastmoleküls stabiler ist als die der „sauren“ Form. Dies führte im Verein mit einigen anderen Beobachtungen zu dem Schluß, daß die Cyclodextrine in ihren Einschlußverbindungen die Rolle der elektrophilen Komponente spielen.

1 2. Teilauszug der am 16. April 1953 der math.-nat. Fakultät der F.U. Berlin eingereichten Habilitationsschrift. 1. Teilauszug vgl. W. Broser u. W. L a u t s ch, Z. Naturforschg. 8 b, 711 [1953].
In der vorliegenden Mitteilung soll die dieser Aussage zugrundeliegende Gl. (9) der vorigen Arbeit, die durch Kombination der einzelnen MWGG. erhalten wurde, auch noch experimentell verifiziert werden. Hierzu werden die $\alpha$ - und $\beta$-Dextrin-Einschlußverbindungen der "alkalischen" und "sauren" Form des Methylorange herangezogen werden. Die hierbei beobachtete, ganz außerordentlich starke Veränderung des Spektrums der „sauren“ Form soll dann näher erörtert werden, ferner wird gezeigt werden, daß im Cyclodextrin-Hohlraum eine hohe Feldstärke herrscht, daß deshalb in ihm polare und polarisierbare Moleküle festgehalten werden, daß die Einschlußverbindung desto stabiler ist, je polarisierbarer das eingeschlossene Molekül ist, und daß der elektrophile Charakter des Cyclodextrins eine Folge hiervon ist. 